\title{
Research on Optimization of Chinese Massage Techniques Course Teaching Mode
}

\author{
Haikuan Wang, Liangbing Yang, Jihong Wang \\ Clinical Medical College of Acupuncture, Moxibustion and Rehabilitation, Guangzhou University of \\ Chinese Medicine, Guangzhou Guangdong, 510405, China
}

Keywords: Chinese massage techniques, Chinese medical course, Teaching mode.

\begin{abstract}
Surveys show that less than one tenth of graduates in acupuncture and massage major from colleges of traditional Chinese medicine are proficient in massage therapy. The basic reason is that most acupuncture and massage majors haven't do enough practice to lay a solid foundation, or are unable to stand high-duty massage because of low fatigue durability due to weak physical ability and have to abandon massage. In view of this, it is suggested to optimize the mode of Chinese massage techniques course teaching from perspective of teaching objectives, teaching contents, course installation, and examination and evaluation, etc., to improve the teaching results of Chinese massage techniques course, and help students to lay a solid physical foundation for massage practice in future and to grasp and apply Chinese massage therapy.
\end{abstract}

\section{Introduction}

Chinese massage techniques is a required course for acupuncture and massage majors of colleges of traditional Chinese medicine. The effect of massage practice has direct influence on students' learning about massage techniques and massage therapy. Thus, massage techniques teaching is important in the massage science. It is an effective way to lay a foundation for students' massage learning by optimizing the teaching mode of massage techniques course to improve the teaching effect. Teaching mode refers to typical stable teaching pattern established under the guidance of certain teaching thought ${ }^{[1]}$ or strategic system or teaching style ${ }^{[2]}$ relating to teaching procedures. Teaching mode mainly consists of teaching thought, teaching objectives, operation process, teachers and students, evaluation, etc ${ }^{[3]}$. This paper is written to discuss methods of improving teaching results of massage techniques course from perspective of teaching objectives, teaching contents, course installation, and evaluation, etc..

\section{Optimize teaching objectives, and get the significance of learning massage techniques across.}

The learning of massage techniques is helpful to enhance fatigue durability and build body, to lay a foundation for technique learning

"To do a good job, an artisan needs the best tools." Without a solid foundation, it is difficult for students to perform massage enduringly, energetically, equably, softly and deeply. The exercises of “eight horses pushing, and nine head of cattle pulling” are helpful to enhance muscular strength, facilitating trembling manipulation and shaking manipulation learning. The exercises of "three ups and downs, and hungry tiger's claw" are helpful to enhance muscular endurance, facilitating continuity and coherence of techniques.

The learning of massage techniques is helpful to enhance the sensitivity and improve the accuracy of clinical diagnosis

Doctors who regularly practice massage techniques and are experienced in clinical massage therapy can accurately point out the focus of clustered nodules, while beginners cannot do it because of unskillfulness and low sensitivity. Most clustered nodules deeply underlie muscles, and are linked with normal muscular tissues, which are difficult to be identified and demanding for skill and sensitivity. The exercises of "Phoenix wings and black dragon displaying its claws" are helpful to 
strengthen technique and sensitivity. Persisting in practice is helpful to raise the accuracy of clinical diagnosis.

The learning of massage techniques is helpful to give rational rehabilitation guidance, to improve the clinical effect

Massage can stimulate the circulation of the blood, cause the muscles and joints to relax, promote flow of qi and blood circulation, improve nutrition supply to soft tissues, and help adhesion lysis, muscles and bones to recover. Take the example of scapulohumeral periarthritis which is a common disease with a long course and tending to recur. The exercises of holding the gate of heaven, plucking a star and exchanging a star cluster, black dragon displaying its claws, and nine ghosts drawing sabers are helpful to mitigate the influence of wind-cold-wetness evil on shoulders, prevent scapulohumeral periarthritis from recurring, relieve the pains, and improve the therapeutic effect for a healed patient.

\section{Optimize teaching contents}

\section{Emphasize massage practice with equipment}

To help students to enhance physical quality and tone up muscles and bones, the teaching of massage techniques in most colleges of traditional Chinese medicine focuses on Yi Jin Jing and Shaolin internal qigon. Yet most students can hardly make standard actions at the beginning because they seldom have physical training. Thus, it is advisable to ask students to make massage practice with equipment before Yi Jin Jing and Shaolin internal qigon. There are four types of massage practice equipment, i.e. strength training equipment such as barbell and dumbbell, sensitivity training equipment such as quincuncial piles and rotating dial, toughness training equipment such as pull ligament, and endurance training equipment such as running machine. Students can make exercises with these equipment to enhance muscular strength, toughness, body flexibility and coordination, and muscular endurance, and then learn Yi Jin Jing and Shaolin internal qigon on this basis, to yield twice the result with half the effort.

\section{Emphasize static exercise}

Through learning Yi Jin Jing and Shaolin internal qigon, students can tone up their muscles, bones, and enhance their strength and muscular endurance, laying a solid foundation for massage technique learning. Static exercise mainly aims to foster internal qi. Through static exercise, students can know better about internal qi and internal force, and control the strength, direction and angle of massage more flexibly, to perform massage enduringly, energetically, equably, softly and deeply. That is, the effect of "once the focus is identified, the focus is accurately located, and skilled techniques are naturally applied thanks to previous practices" as specified in the Golden Mirror of Medicine is achieved.

\section{Emphasize the link between massage therapy and clinical rehabilitation}

Massage has been being an important means of health cultivation and rehabilitation since ancient times, helpful to stimulate the subjective initiative of patients and promote disease rehabilitation. Rehabilitation is a development tendency and difficulty of modern medicine, which can be overcome via massage. Only by applying massage to rehabilitation medicine, massage can be revitalized.

\section{Optimize teaching method to arouse students' enthusiasm}

\section{Combine teachers' demonstration and multimedia teaching}

Most students are liable to be distracted. Thus, applying multiple teaching methods is helpful to promote students' thinking activities, keep students excited, and then make students concentrate ${ }^{[4]}$. Teachers' demonstration refers to that teachers show the concrete operations to students first, then guide students to practice by pausing a while after doing each action, and correct students' nonstandard actions. The method of combining personal examination with verbal instruction is of great importance. Take the example of the exercise of hungry tiger's claw. Teacher can show this action in an evolutionary way, standing on the ground with palms, then with the five fingers, and last with three fingers. Thus, students can know about the teacher's force and skill. Then, students can 
practice as the teacher did. Besides, multi-media classroom can be made use of to illustrate the functions, characteristics, considerations and historical origin of actions, to help students to learn and grasp.

\section{Enhance exercise after class to improve teaching quality}

It is advisable to attentively help students with weak foundation by correcting their wrong actions or gestures, and learning about their feelings and experiences. Besides, teachers themselves should be proficient enough in massage and patient, have sufficient time for partner training, and make scientific instruction scheme ${ }^{[5]}$. Excellent students can be assigned to help and instruct their inferior mates after class, attendance rate can be made use of to supervise and urge students to do exercise, teaching videos can be provided to students for their imitating practice after class, and massage competition (focusing on Yi Jin Jing and Shaolin internal qigon) can be regularly held to arouse students' enthusiasm and improve teaching quality.

Concentrate on clinical application of massage, combine massage and clinic, and stress the practicability of massage, to arouse students' enthusiasm

Massage can be used for revalidation, health cultivation and body building. Massage techniques make massage therapy featured by active exercise. Exercise is one of the main methods of treating diseases relating to spine, and also promotes the recovery of chronic diseases. Before teaching massage techniques, teachers can explain clinical application of techniques, so that students can know better about the clinical application value of techniques. For example, the exercise of plucking a star and exchanging a star cluster is specific to dorsal and lumbar muscles, levator ani, front and back muscles of lower limbs, etc., helpful to enhance waist force, leg strength, and treat soreness and weakness of waist and knees, and impotence and premature ejaculatioin; the exercise of nine ghosts drawing sabers is specific to cervical muscle, triceps muscle of arm, bicipital muscle of arm, levator scapulae, trapezius, latissimus dorsi, forearm flexor groups, etc., helpful to enhance muscle force of neck and arm, and treat cervical spondylosis, scapulohumeral periarthritis, degeneration of shoulder and back, etc. In this way, students can understand better about the clinical application and the practicability of techniques, and then increase interest in techniques learning.

\section{Optimize evaluation method}

\section{Repeatedly organize examination to supervise and urge students to do exercise}

The practical operation examination of massage techniques can be divided into stages by every four or five moves, and evaluated based on students' performance, which should be taken as a part of the total results. Thus, students will become more active in practice, and the teacher can learn about students' learning state in time so as to point out and correct the defects. Besides, the attendance rate of practice after class also can be included in the total results, to urge students to take exercise. It is feasible to promote students to persist in exercise by organizing techniques examination at the beginning of each semester, to prevent students from slacking in holidays.

\section{Bear in mind classics on massage techniques, and grasp technique essentials}

The text of Yi Jin Jing covers the essentials and considerations of twelve moves. The second move of hsiang mo ch'u: "lead toe down to the ground, stretch both hands levelly forward; relax, gapemouthed”. Read this sentence carefully over and over again, and you can grasp this move and do it well: lead toe down to the ground, stabilize the bottom, stretch arms sidewards and rise as high as the shoulder, calm, gently breathe, contemplate, meditate, slightly close mouth, widely open eyes to brim with radiating vigour, and look at the front horizontally. The exercise of this move is helpful to enhance cardio-pulmonary function.

Strengthen theoretical examination, and combine theory with clinical practice, to realize practice on the spot

Theoretical examination mainly covers the clinical application of massage techniques to common diseases, which urges students to keep in mind the functions of every moves of massage techniques to give proper instructions to patients in future. 
Through optimization of teaching objectives, teaching methods, teaching contents and evaluation methods, teachers will turn to be concerned about students' needs to delivery lessons in a visual, vivid and flexible way, so as to arouse students' enthusiasm and improve teaching effect. Instructing students' to do physical exercise can help students to enhance their muscle fatigue durability, to lay a solid physical foundation for clinical practice in future. Besides, since massage therapy involves no medicine and trauma, more and more attention will be paid to it. "To boom massage, techniques must be emphasized; to emphasize techniques, exercises must be made"[6]. Exercises for massage are an important part of massage science, which can help massage practitioners to grasp essentials of moves and gestures to train finger force, arm strength, waist force and leg strength. Through these exercises, together with breathing training and change, massagist will become strong enough to handle massage therapy ${ }^{[7]}$. Therefore, the learning and research of clinical application of massage techniques should be strengthened, to help acupuncture and massage majors to get improved.

\section{References}

[1] Yu Deshen, Zhu Xuesi. Exploration in New Teaching Mode, Shandong Education Research,1989, (4):31-33.

[2] Liu Haimin. Discussion on Teaching Mode., Journal of the Chinese Society of Education,1988, (5):34-37.

[3] Hao Zhijun, Xu Jixue. 20-year History of Research on Teaching Mode 20: Course, Issues and Direction, Theory and Practice of Education, 2003, 23(12):51-55.

[4] Meng Qian, Tian Qiwu, Zan Tao. Discussion on Method of Massage Techniques Examination, Journal of Jianghan University: Medical Science, 2002, 30(4):45-46.

[5] Zhang Guanzhong, Lu Yangjia, Huang Yong. Discussion on Excellent Course Construction of Massage Techniques, Hebei Journal of Traditional Chinese Medicine, 2012, 34(5):796-798.

[6] Liao Jun, Wang Shizhong, Xu Teng. Thinking on Massage Teaching Reform, Journal of Sichuan College of Education,2008,24(6):29-30.

[7] Peng Xuming. Comments on Massage Techniques Teaching, Chinese Manipulation \& Qi Gong Therapy,2007, 24(3):7-8. 